\title{
Early Prediction of Non-Cardiac disorders From ECG Using Lab view
}

\author{
Purnima.S, Aditya.S, Meenakshi.E, Narumugai.L, Yamini.E
}

\begin{abstract}
The Electrocardiogram (ECG) is one of the most basic cardiological test done for any suspected diseases related to cardiological system. Abnormalities in any other system can also be detected with change in morphology of ECG. In this paper we note the changes in morphology of ECG for prediction of non-cardiac diseases like Emphysema, CNS haemorrhage, Thyroidism, Hypokalemia and Hyperkalemia. ECG is used to predict these diseases as it is a non-invasive technique and also the morphology of ECG wave is repetitive until any abnormality manifests itself through ECG. If any of the above mentioned non-cardiac diseases occur, significant changes appear in ECG signal and with the knowledge of these changes, early clues are provided regarding the diseases which are lifesaving. This paper works on acquisition and segmentation of ECG for extraction of features that are inevitable for the prediction of above mentioned diseases. The extracted features are classified as normal or abnormal based on the comparison with the reference signal. The reference signal contains information about the normal and abnormal morphological conditions of ECG which are segmented, extracted and stored prior in the LabVIEW. The automatic prediction of non-cardiac diseases is carried out with LabVIEW through which a tolerance method is used to correctly compare and predict that particular kind of disease. This will be later extended to real-time acquisition, processing and classification. The basic motive behind this project is to create an awareness and alert the patient before the fatal stage.
\end{abstract}

Index Terms: ECG, LabVIEW

\section{INTRODUCTION}

Many diseases in human body doesnot show any early symptoms, which may become a fatal condition for the human later on. Likewise there are some non cardiac diseases which doesnot show significant symptoms in the body but has a changes in ECG from heart. Through this project, it is easier to diagonis the diseases in the earlier stage to avoid a fatal condition in which the person is being pushed.

Revised Manuscript Received on July 22, 2019.

Purnima.S, Asst.Professor, Department of Biomedical Engineering,Jerusalem College of Engineering, Pallikaranai, Chennai-600 100, INDIA.

Aditya.S, UG Scholar, Department of Biomedical Engineering,Jerusalem College of Engineering, Pallikaranai, Chennai-600 100, INDIA.

Meenakshi.E, UG Scholar, Department of Biomedical Engineering,Jerusalem College of Engineering, Pallikaranai, Chennai-600 100, INDIA.

Narumugai.L, UG Scholar, Department of Biomedical Engineering,Jerusalem College of Engineering, Pallikaranai, Chennai-600 100, INDIA.

Yamini.E, UG Scholar, Department of Biomedical Engineering,Jerusalem College of Engineering, Pallikaranai, Chennai-600 100 , INDIA.

\section{NON-CARDIAC DISEASES RELATING TO ECG}

\section{(i)Emphysema :}

Emphysema is a disease of the lungs. It is a type of chronic obstructive pulmonary disease (COPD) and involves the loss of elasticity and enlargement of the air sacs in the lung. The alveoli are the areas of the lung where oxygen and carbon dioxide are exchanged into and out of the blood, which is present at the end of the bronchioles of the lung become enlarged because their walls break down or the air sacs are destroyed, narrowed, collapsed, stretched, or over-inflated. Having fewer and larger damaged sacs means there is a reduced surface area for the exchange of oxygen into the blood and carbon dioxide out of it. The damage is permanent. The ability to breathe properly cannot be fully recovered.

\section{\# Effects on ECG -}

- The presence of increased air between heart and recording electrodes has a damping effect, leading to reduced amplitude of the QRS complexes.

- Absence of R wave and depressed $\mathrm{Q}$ wave is due to downward displacement of diaphragm resulting in vertical heart position.

(ii) Thyroidism :

The thyroid gland influences almost all of the metabolic processes in your body. Thyroid disorders can range from a small, harmless goitre (enlarged gland) that needs no treatment to life-threatening cancer. The most common thyroid problems involve abnormal production of thyroid hormones. Too much thyroid hormone results in a condition known as hyperthyroidism. Insufficient hormone production leads to hypothyroidism.as shown. Although the effects can be unpleasant or uncomfortable, most thyroid problems can be managed well if properly diagnosed and treated.

\section{\# Effects on ECG -}

- TSH level were marginally associated with QRS interval. Higher TSH levels show inverted T wave.

- Bradycardia is well known signs of hypothyroidism. Slower heart rate is due to the effects of thyroid hormone on sodium pump density and enhancement of $\mathrm{Na}^{+}$and $\mathrm{K}^{+}$ currents.

$$
\begin{aligned}
& \text { Blue Eyes Intelligence Engineering } \\
& \text { \& Sciences Publication }
\end{aligned}
$$

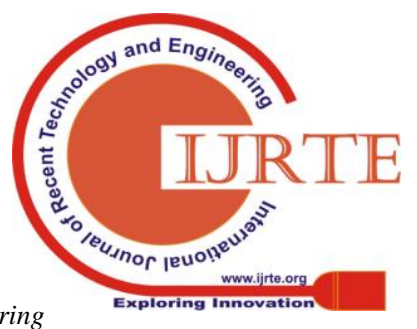




\section{Early Prediction Of Non-Cardiac Disorders From Ecg Using Labview}

- Tachycardia is well known signs of hyperthyroidism. Increased heart rate is due to the effects of thyroid hormone on sodium pump density and enhancement of $\mathrm{Na}^{+}$and $\mathrm{K}^{+}$ currents.

\section{(iii) Hypokalemia :}

Hypokalemia is a low level of potassium $(\mathrm{K}+)$ in the blood serum. Potassium is an important electrolyte for nerve and muscle cell functioning, especially for muscle cells in the heart. Kidneys control body's potassium levels, allowing for excess potassium to leave the body through urine or sweat. Normal potassium levels are between 3.5 and $5.0 \mathrm{mmol} / \mathrm{L}$ with levels below $3.5 \mathrm{mmol} / \mathrm{L}$ defined as hypokalemia.

\section{\# Effects on ECG -}

- Sagging of ST segment, depression of T wave.

- Elevation of the U wave and short PR interval.

\section{(iv) Hyperkalemia :}

Hyperkalemia is an elevated level of potassium $(\mathrm{K}+)$ in the blood serum. Normal potassium levels are between 3.5 and $5.0 \mathrm{mmol} / \mathrm{L}$ with levels above $5.5 \mathrm{mmol} / \mathrm{L}$ defined as hyperkalemia. An abnormal heart rate can occur which can result in cardiac arrest and death. Common causes include kidney failure, hypoaldosteronism, and rhabdomyolysis.

\section{\# Effects on ECG -}

- Bradycardia due to viscous cycle in the setting of medications.

- Renal failure- accumulation of some AV node blockers.

- Suppresses impluses from AV, SA leading to cardiac arrest.

\section{(v) CNS Hemorrhage :}

CNS Hemorrhage, also known as cerebral bleed, is a type of intracranial bleed that occurs within the brain tissue or ventricles. Since the brain cannot store oxygen, it relies upon a series of blood vessels to supply oxygen and nutrients. When a bleed occurs, oxygen may no longer be able to reach brain tissue supplied by leaky or broken blood vessels. Pooling of blood from an intracranial bleed or CNS bleed also puts pressure on the brain and deprives it of oxygen. When a bleed or stroke interrupts blood flow around or inside the brain, depriving it of oxygen for more than three or four minutes, the brain cells die. The affected nerve cells and the related functions they control are damaged as well.

\section{\# Effects on ECG -}

- Cerebral T waves with short QT interval. occurs.

- ST segment depression and elevated catecholamine levels is related to QT interval short.
- T wave inversion will be for years and bradycardia

\section{METHODOLGY}

In this project we acquired the signal from patients using ECG and processed it in LabVIEW.

\section{(i) ECG :}

Electrocardiography is the process of recording the electrical activity of the heart over a period of time using electrodes placed on the skin and the normal ECG is shown in fig 1 . These electrodes detect the tiny electrical changes on the skin that arise from the heart muscle's electrophysiologic pattern of depolarizing and repolarizing during each heartbeat. It is a very commonly performed cardiology test. In a conventional 12-lead ECG, ten electrodes are placed on the patient's limbs and on the surface of the chest.

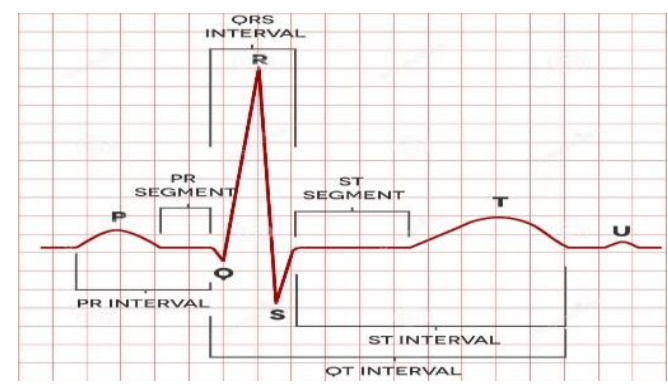

Fig 1. Normal ECG

To acquire significant changes in ECG caused by these diseases, acquiring signal from chest was more prominent and its position is shown in fig 2. Placement of electrodes on chest:

- V1 - Fourth intercostal space on the right sternum

- V2 - Fourth intercostal space at the left sternum

- V3 - Midway between placement of V2 and V4

- V4 - Fifth intercostal space at the midclavicular line

- V5 - Anterior axillary line on the same horizontal level as V4

- V6 - Mid-axillary line on the same horizontal level as V4 and V5

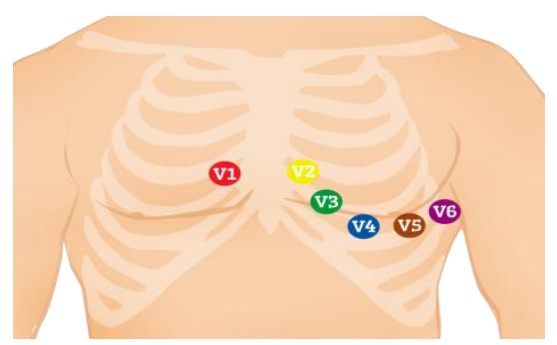

Fig 2. Pericardial Electrode Placement

\section{(ii) LabVIEW}

LabVIEW (Laboratory Virtual Instrument Engineering Workbench), created by National Instruments (www.ni.com) is a graphical programming language that uses icons instead of lines of text to create applications.

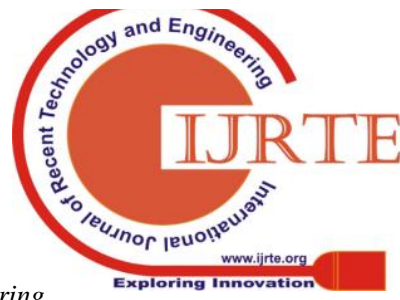


LabVIEW programs/codes are called Virtual Instruments, or VIs for short. LabVIEW is used for Data acquisition, signal Processing (Analysis), and hardware control - a typical instrument configuration based on LabVIEW.

The signals from the patients are acquired with the help of ECG sensor and DAC helps to transfer the signal from the sensor to the system that is to the LabVIEW, which is shown in fig 3. The signals are read one after the other as per the disease in the file and are displayed using a graph indicator. Then the signals are filtered by using a wavelet denoise block and is sent to the feature extraction block. Meanwhile the signals are sent to the array size and amplitude measurement block in order to find out the size of the signal and the positive and negative peaks of the signals. Now using the help of a ECG feature stats block all the important and vital features of the ECG are extracted and are saved. In the meantime, the denoised signal is given to a HRV analysis pallet and the R-R intervals are calculated. In order to classify the disease's, the features are compared and clustered with the help of comparison tools. These values are kept as a constant value and the signals acquired in real time are made to go through the same process and their features are compared with the derived constant/standard values of all the diseases. The full block diagram for acquisition and analysis of ECG signal is shown in fig 3.This process is done by using a comparison function and a case structure. The features are compared, if all the criteria are satisfied then the case structured will display the disease associated to those features thereby classifying and predicting the disease.

The complete VI setup of the working is shown in fig 5.

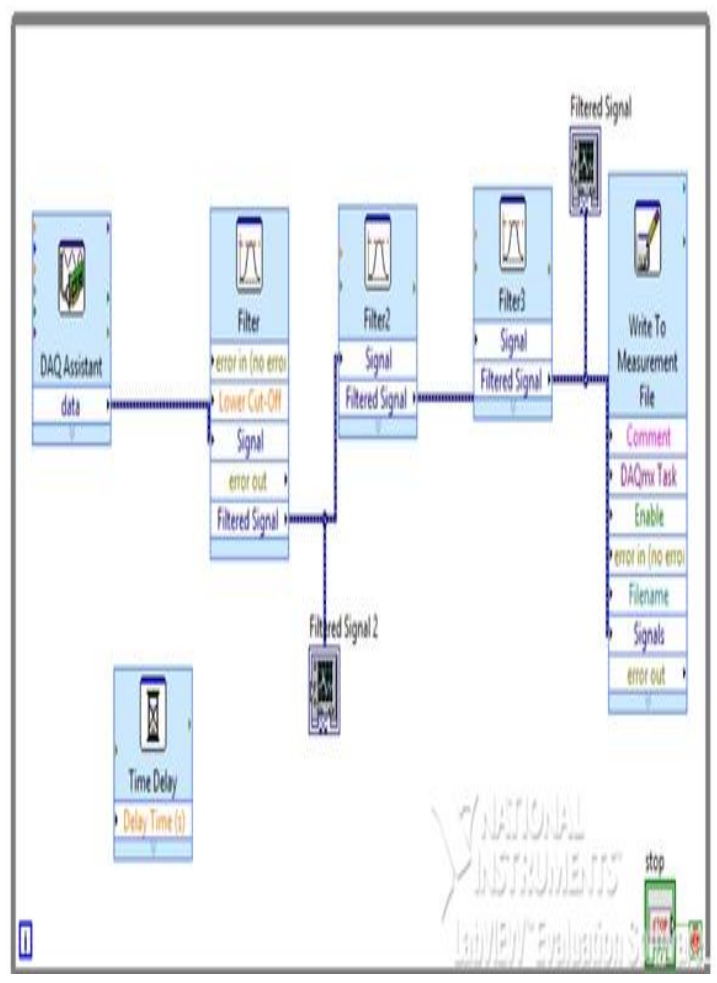

Fig 3. Block Diagram of Data Acquisition

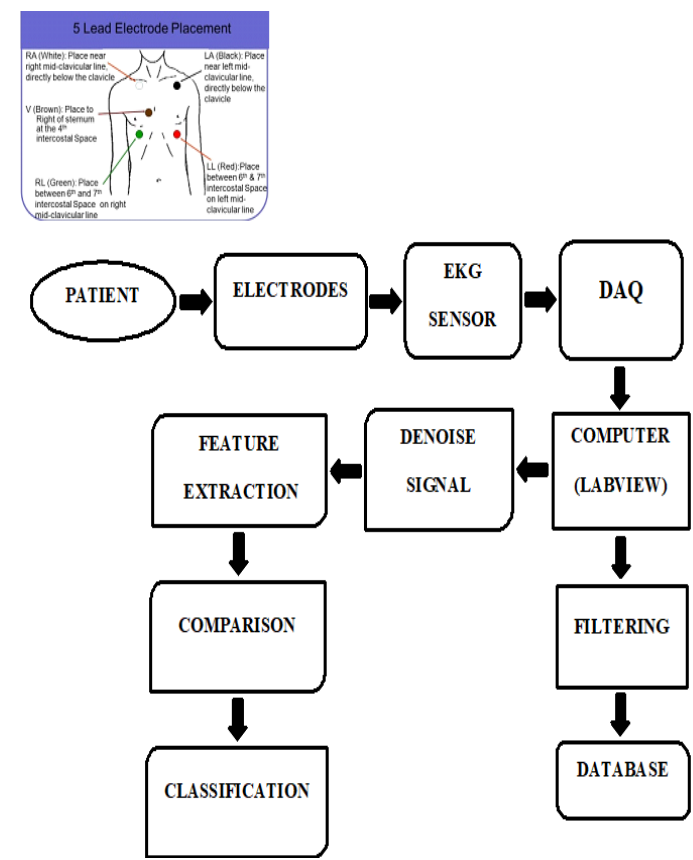

Fig 4. Block Diagram for Acquisition and Analysis of ECG Signal



Fig 5. Block Diagram of VI in LabVIEW

\section{RESULTS AND DISCUSSION}

We collected five data for each diseased condition and extracted the features from all the signals. By extracting the features from the signal we were able to classify the signals. Normal values for waves and intervals are as follows:

- RR interval: 0.6-1.2 sec

- P wave: $80 \mathrm{~ms}$

- PR interval: 120-200 ms

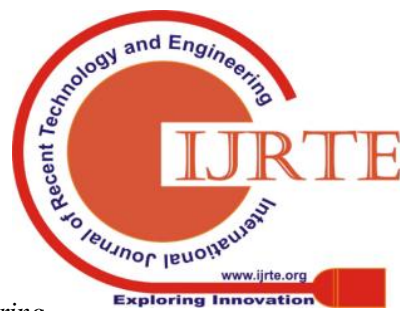


Early Prediction Of Non-Cardiac Disorders From Ecg Using Labview

- PR segment: 50-120 ms

- QRS complex: 80-100 ms

- ST segment: 80-120 ms

- T wave: $160 \mathrm{~ms}$

- ST interval: $320 \mathrm{~ms}$

- QT interval: $420 \mathrm{~ms}$

\begin{tabular}{|c|c|c|}
\hline FEATURES & $\begin{array}{c}\text { STANDARD } \\
\text { VALUE }\end{array}$ & WAVE 1 \\
\hline QRS std (mv) & $<0.02$ & 0.018 \\
\hline $\begin{array}{c}\text { QRS } \\
\text { amplitude } \\
\text { mean (mv) }\end{array}$ & $>1$ & 1.12 \\
\hline $\begin{array}{c}\text { Depressed Q } \\
\text { wave (-ve) } \\
\text { (mv) }\end{array}$ & $<1.5$ & 1.7 \\
\hline \multicolumn{2}{|c|}{}
\end{tabular}

Table 1. Feature extracted from Emphysema

Table 1 shows the features of emphysema disease. It shows the change in QRS mean, QRS amplitude and all the other features.

\begin{tabular}{|c|c|c|}
\hline FEATURE & $\begin{array}{c}\text { STANDARD } \\
\text { VALUE }\end{array}$ & WAVE 1 \\
\hline $\begin{array}{c}\text { Inverted T } \\
\text { wave(mv) }\end{array}$ & $>-1.5$ & -1.4505 \\
\hline $\begin{array}{c}\text { ST segment } \\
\text { depression (s) }\end{array}$ & $>-1$ & -0.1445 \\
\hline $\begin{array}{c}\text { Short QT } \\
\text { interval (s) }\end{array}$ & $<0.200$ & 0.1490 \\
\hline $\begin{array}{c}\text { Reduced R wave } \\
\text { (mv) }\end{array}$ & $<1$ & 0.6780 \\
\hline
\end{tabular}

Table 2. Feature extracted from CNS

\section{Hemorrhage}

Table 2 shows the features of CNS hemorrhage disease. It shows the change in QT interval, $\mathrm{R}$ wave and all the other features.

\begin{tabular}{|c|c|c|}
\hline FEATURES & $\begin{array}{c}\text { STANDARD } \\
\text { VALUES }\end{array}$ & WAVE 1 \\
\hline Inverted T wave & $<-1$ & -1.1800 \\
\hline $\begin{array}{c}\text { QRS } \\
\text { voltage(mv) }\end{array}$ & $<2$ & 1.1555 \\
\hline $\begin{array}{c}\text { Tachycardia } \\
\text { (bpm) }\end{array}$ & $>250$ & 286 \\
\hline $\begin{array}{c}\text { ISO level } \\
\text { (bol }\end{array}$ & $>0.5$ & 0.787 \\
\hline
\end{tabular}

Table 3. Features extracted from Thyroidism

Table 3 shows the features of thyroidism disease. It shows the change in QRS voltage, ISO level and all the other features.

\begin{tabular}{|c|c|c|}
\hline FEATURES & $\begin{array}{c}\text { STANDARD } \\
\text { VALUE }\end{array}$ & WAVE 1 \\
\hline $\begin{array}{c}\text { Peaked T } \\
\text { wave(mv) }\end{array}$ & $>1.5$ & 1.5 \\
\hline $\begin{array}{c}\text { Depressed S } \\
\text { wave(mv) }\end{array}$ & $<-1$ & -1.9 \\
\hline $\begin{array}{c}\text { Low QRS } \\
\text { interval(s) }\end{array}$ & $<0.1$ & 0.02 \\
\hline
\end{tabular}

Table 4. Features extracted from Hyperkalemia

Table 4 shows the features of hyperkalemia disease. It shows the change in QRS interval, $\mathrm{S}$ wave and all the other features.

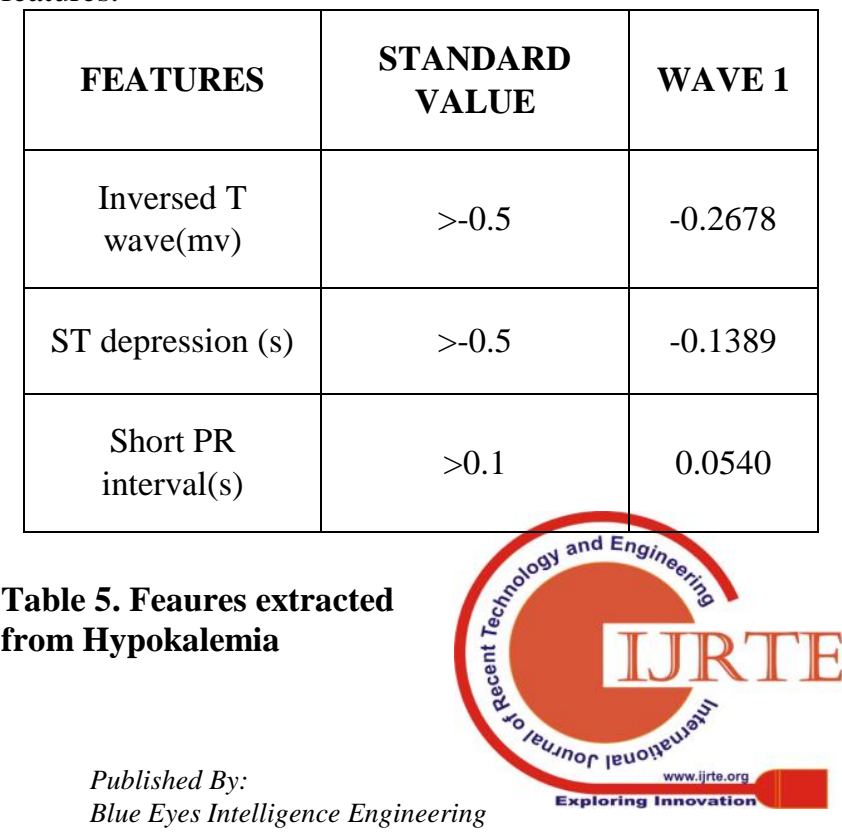


Table 5 shows the features of hypokalemia disease. It shows the change in PR interval, $\mathrm{T}$ wave and all the other features.

\section{CONCLUSION AND FUTURE WORK}

\section{Conclusion}

Thus, by obtaining the features of ECG we are able to classify the disease. We can detect the disease for any patients. since ECG is one of the most common laboratory test taken. We can also detect the changes in patients who are continuously monitored through ECG. This can be later extended to real-time acquisition, processing and classification. The basic motive behind this project is to create an awareness and alert the patient before the fatal stage.

\section{Future work}

- More features can be extracted from the ECG.

- Same process can be extended from more non-cardiac diseases.

- The signals can be made to transfer through telemetry.

- Constant monitoring can be done if the telemetry is made compact and handy.

\section{REFERENCES}

1. ECG in non cardiac disorders - journal - Yash lokhandwala, Pallavi lanjewar, Sameer Ambar (2005)

2. LabVIEW Based Real Time Bio-Telemetry System for Healthcare -journal-Jay A. Raval, Vivek V. Sakinala, Nitin R. Jadhav and Deepak C. Karia(April 2017)

3. An ECG Patch Combining a Customized Ultra-Low-Power ECG SoC with Bluetooth Low Energy for Long Term Ambulatory Monitoring-journal-Marco Altini, Salvatore Polito, Julien Penders, Hyejung Kim, Nick Van Helleputte, Sunyoung Kim, Firat Yazicioglu

4. Cardiac Abnormalities in Subarachnoid Hemorrhage - A ResumeBernard M. Weintraub and Lawrence C. McHenry (2017)

5. Thyroid Hormones and Electrocardiographic Parameters: Findings from the Third National Health and Nutrition Examination SurveyYiyi Zhang, Wendy S. Post, Alan Cheng, Elena Blasco-Colmenares, Gordon F. Tomaselli, Eliseo Guallar (2013)

6. ECGDiagnosis: Hyperkalemia - journal - Perm J (2013)

7. Rajesh, M., and J. M. Gnanasekar. "Path Observation Based Physical Routing Protocol for Wireless Ad Hoc Networks." Wireless Personal Communications 97.1 (2017): 1267-1289.

8. N. Nithiyanandam, K. Venkatesh, M. Rajesh, Transfer The Levels Of The Monitored Carbon, Nitrogen Gases From The Industries, International Journal of Recent Technology and Engineering, Volume-7 Issue-6S3 April, 2019.

9. Sivanesh Kumar, A., Brittoraj, S., Rajesh, M., Implementation of RFID with internet of things, Journal of Recent Technology and Engineering, Volume-7 Issue-6S3 April, 2019.

10. Rajesh, M., Sairam, R., Big data and health care system using mlearningJournal of Recent Technology and Engineering, Volume-7 Issue-6S3 April, 2019.

11. ECG Diagnosis: Hypokalemia - journal - Perm J (2013)

\section{AUTHORS PROFILE}

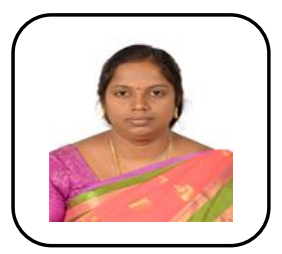

Ms. S. Purnima is currently working as an Assistant Professor, in the Department of Biomedical Engineering, Jerusalem College of Engineering, Chennai. She is also pursuing her $\mathrm{Ph} . \mathrm{D}$ in Functional MRI analysis studies in Anna University, Chennai, India. She completed her M.E in Medical Electronics from Anna University, Chennai, Tamil Nadu in 2006 and B.E in ECE from St. Joseph's College of Engineering, Chennai, Tamil Nadu, India in 2003. She has 10 years of Teaching Experience in Biomedical Engineering. She published many national and international conference and journal papers in Scopus indexed and UGC indexed journals. Her research interests are Rehabilitation Engineering and Biomedical instrumentation. She is a life member of the Indian society for Technical Education, and Biomedical Engineering Society of India. 